\title{
Tubulo-villous adenoma with severe dysplasia in the prostatic urethra: A case report and histogenetic considerations
}

\author{
Tadashi Terada * \\ Department of Pathology, Shizuoka City Shimizu Hospital, Shizuoka, Japan
}

Received: March 22, 2016

DOI: $10.5430 /$ crcp.v4n1p22
Accepted: September 20, $2016 \quad$ Online Published: October 14, 2016

URL: http://dx.doi.org/10.5430/crcp.v4n1p22

\begin{abstract}
In 2002, Seibel et al. presented a case series of 18 patients with villous adenomas of the urethra. Since then, only a couple of reports regarding this rare entity have been published. Recently (2013), Kao and Epstein proposed a new entity of tubular adenoma (4 cases) in the urinary tract, being similar to tubular adenomas in gastrointestinal (GI) tract; no following cases have been reported. Herein presented a case of tubule-villous adenoma (TVA) in the prostatic urethra (PU) of a 77-year-old man male patient suffering from dysuria. Cystscopy showed a polypoid/villous tumor of the PU, and transurethral resection of this tumor was performed. Grossly, fragments of the tumor measured $12 \mathrm{~mm}$ in diameter and polypoid/villous. Microscopically, it was a TVA indistinguishable from GI adenomas. No urothelium was seen, but a few normal prostatic glands were noted. The tumor showed mild to severe dysplasia, but in situ malignant transformation and invasive malignancy were not seen. Histochemically, acidic mucins were present. Immunohistochemical staining results of the tumor cells were as follows: cytokeratin(CK) AE1/3+++, CKCAM5.2+++, CD34BE12+, CK5+, CK6+, CK7++, CK14-, CK20+, EMA+, CDX-2-, CEA++, CA19-9++, CA125-, vimentin-, NSE-, NCAM-, synaptophysin-, chromogranin-, E-cadherin+++, beta-catenin+++, MUC1++, MUC2-, MUC5AC++, MUC6+, PSA+++, AMACR+++, AFP-, HepPar1-, p53-, KIT-, PDGFRA+, MET+, HER2-, Ki67+ (labeling = 5\%). Myoepithelial cells were present in some areas. These results suggest the development and presence of a TVA in the PU indistinguishable from those in the GI tract, and it can arise from prostatic epithelium.
\end{abstract}

Key Words: Adenoma, Tubulo-villous, Urethra

\section{INTRODUCTION}

In 2002, Seibel et al. ${ }^{[1]}$ presented a case series of 18 patients with villous adenomas of the urethra. Since then, only a couple of reports regarding this rare entity have been published. Recently (2013), Kao and Epstein ${ }^{[2]}$ proposed a new entity of tubular adenoma (4 cases) in the urinary tract, being similar to tubular adenomas in gastrointestinal (GI) tract; no following cases have been reported. In the recent report the author presents a rare case of tubulo-villous adenoma (TVA) in the prostatic urethra (PU), which is histologically indistinguishable from adenoma of the GI tract, with interesting immunohistochemical (IHC) results.

\section{CaSe report}

A 77-year-old male Japanese patient presented with dysuria. A cystscopy was performed, showing a polypoid/villous tu-

\footnotetext{
*Correspondence: Tadashi Terada, MD, PhD; Email: piyo0111jp@yahoo.co.jp; Address: Department of Pathology, Shizuoka City Shimizu Hospital, Miyakami 1231 Shimizu-Ku, Shizuoka 424-8636, Japan.
} 
mor of the PU, and subsequently a transurethral resection (TUR) was conducted. Grossly, the TUR fragments of the tumor measured $12 \mathrm{~mm}$ in diameter and polypoid/villous morphology. Microscopically, it was a TVA indistinguishable from GI adenomas (see Figure 1). The epithelium was tall columnar and contained mucins in the cytoplasma as well as in lumens. No Paneth cells were seen, but goblet cells were recognized. No urothelium was seen, but a few normal prostatic glands were noted under the tumor. The tumor cells showed mild to severe dysplasia, but obvious in situ malignant transformation and invasive malignancy were not seen. No lymphatic or venous permiations by tumor cells were observed.
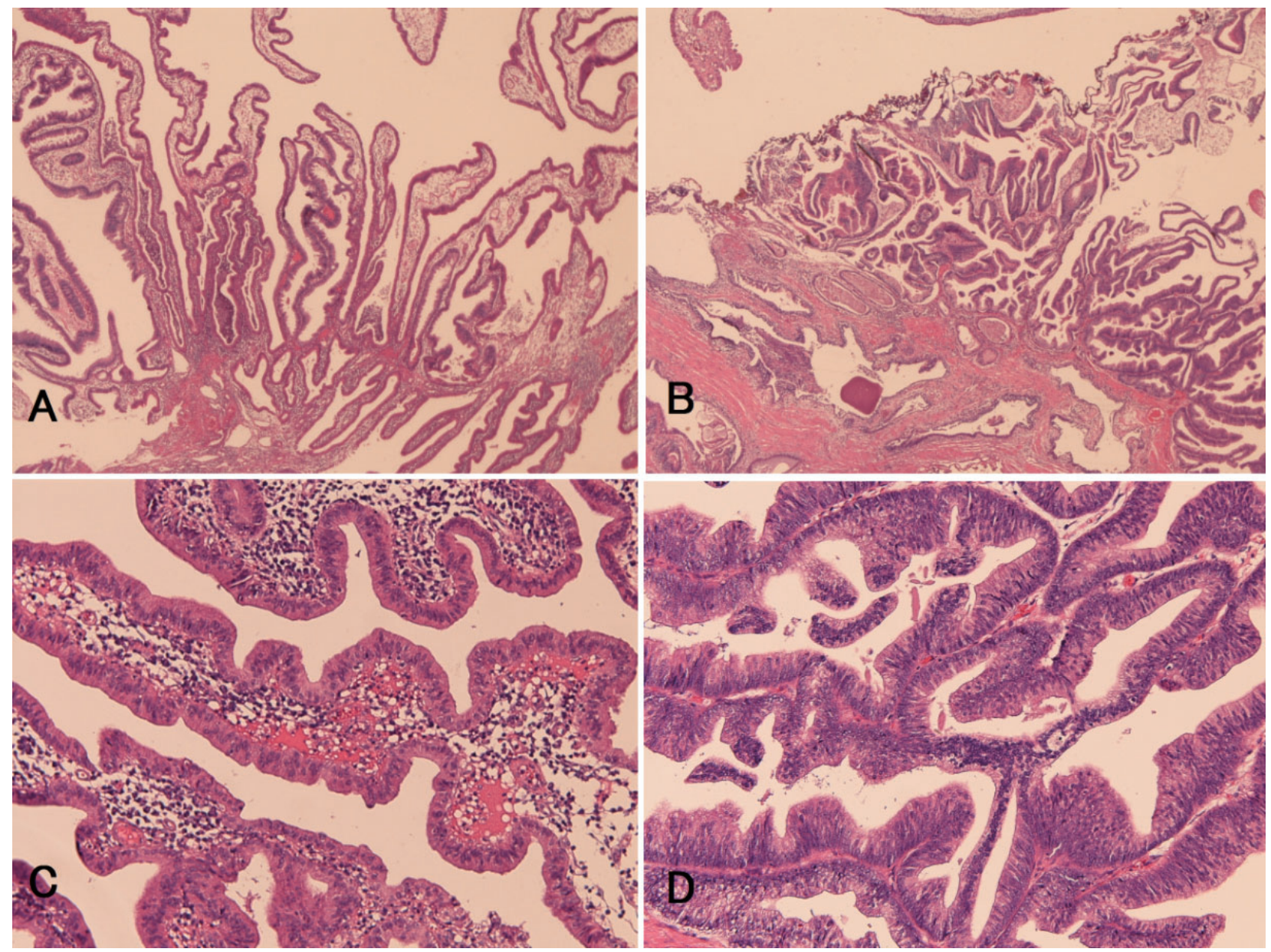

Figure 1. Microscopic features of the tumor in prostatic urethra

The degree of atypia raned from mild $(B)$ to severe $(D)$. Villious/ tubular proliferations of columnar colonic type epithelium is seen. Microscopic features of low power view ( $A$ and $C$ ) and high power view (B and D). Villous pattern predominates in some areas ( $A)$, but tubular pattern is dominant in other areas $(C)$. The degree of dysplasia ranged to mild $(B)$ to severe $(D)$. No invasive malignancy is seen. The glands of the lowermost part of $C$ can be prostatic glands. A, C: $\times 40$. B and D: $\times 150$.

Histochemically (HC), acidic mucins were present, but neutral mucins were absent. IHC results of the tumor cells were as follows: cytokeratin (CK) AE1/3 +++, CKCAM5.2+++, CD34BE12+, CK5+, CK6+, CK7+++ (see Figure 2A), CK14-, CK20+, EMA+, CDX-2-, CEA++ (see Figure 2B), CA19-9++, CA125-, vimentin-, NSE-, NCAM-, synaptophysin-, chromogranin-, MUC1++ (see Figure 2C), MUC2-, MUC5AC++ (see Figure 2D), MUC6+, PSA+++ (see Figure 2E), AMACR+++ (see Figure 2F), by cystscopy once per two months.
E-cadherin+++ (see Figure 2G), beta-catenin+++, AFP-, HepPar1-, p53-, KIT-, PDGFRA+, MET+, and HER2-, Ki67+ (labeling $=8 \%$ ) (see Figure 2I). Myoepithelial cells as revealed by IHC for CK34BE12, CK14, p63, CK5, CK6 were present in some areas of tumor beneath the tumor cells (see Figure 2H). The patient is now free from tumor 5 months after the diagnosis. The patient is carefully followed up by clinical cytology of voided urine two times per month and 


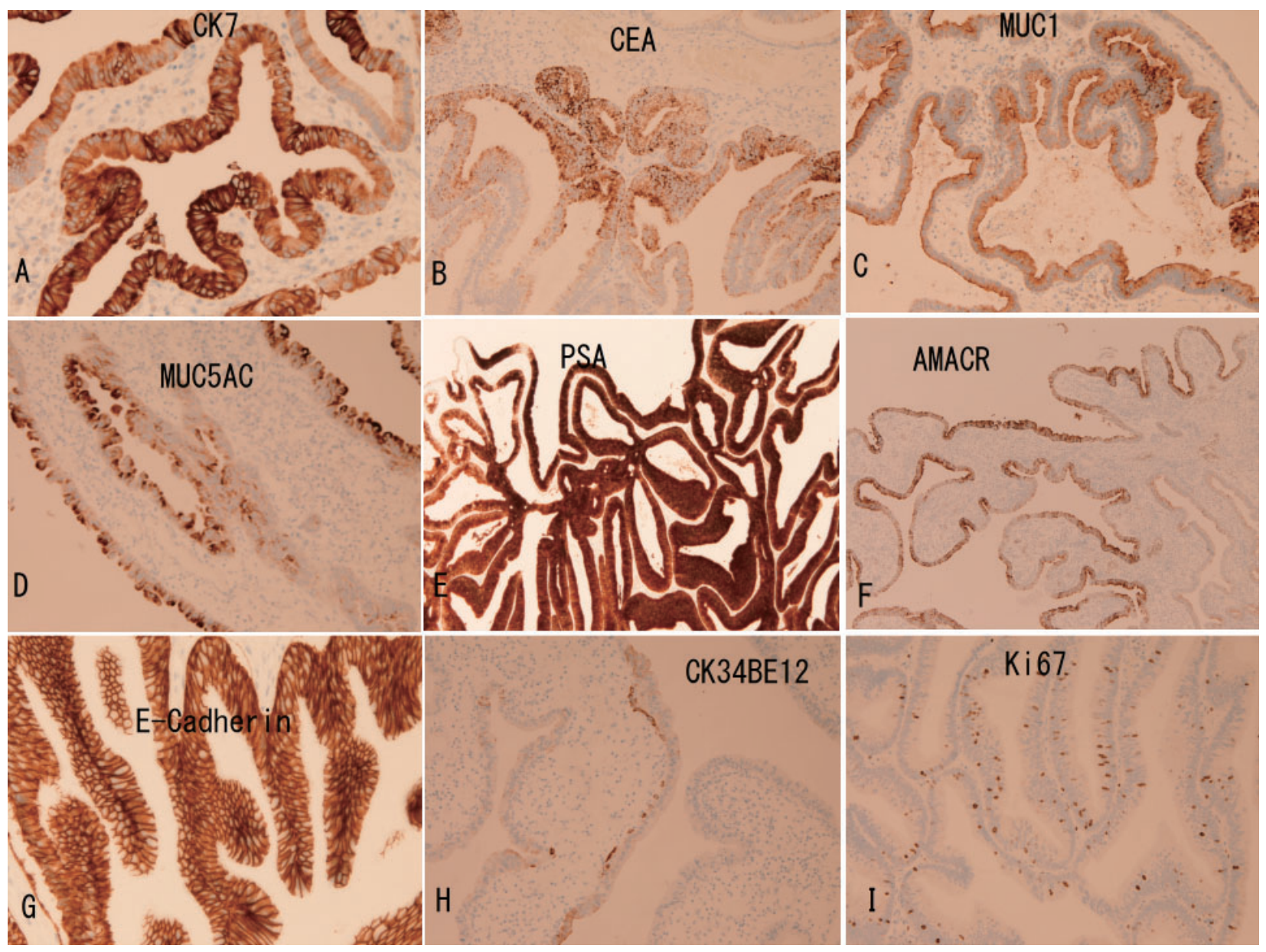

Figure 2. Immunohistochemical features of the tumor

The tumor cells are positive for cytokeratin (CK) $7(A), C E A(B), M U C 1(C), M U C 5 A C(D), P S A(E), A M A C R(F), E-c a d h e r i n(G)$, and Ki67 (labeling index $=8 \%)(I)$. Myoepithelial cells are seen beneath the tumor cells in a minor lesion $(H)$. H: Immunostaining for

CK34BE12. A-D, G: $\times 150 . E, F: \times 80 . H, I: \times 100$

\section{Discussion}

To the best of the author's knowledge, the present case represents the first report of TVA in the urinary tract. The HE histology of the tumor closely resembled TVA of GI tract, in particular colonic one. The HE histology did not suggest urothelial and prostatic origin. The mucus HC suggested acidic mucins and therefore colonic phenotype. The MUC profile suggested gastric phenotype. CEA and CA19-9 suggested GI phenotype. CDX-2 negative pattern did not indicate GI pattern. The neuroendocrine molecules (NCAM, NSE, synaptophysin, chromogranin) did not reveal endocrine cells. E-cadherin and beta-catenin pattern suggested strong adhesion of tumor cells and therefore a lack of invasive malignancy. The data of p53 and Ki67 suggested no p53 mutations and benign proliferative fraction of tumor cells. Lack in tumor cells of high-molecular weight cytokeratin (HMWCK) and lack of p63 suggested no urothelial or squamous nature of tumor cells. The expression of receptor thyrosine kinases
(MET, PDGFRA, KIT, HER2) suggested that HGF/MET and PDGF/PDGFRA may be operative in growth of the tumor but STF/KIT and bFGF/HER pathways not. KIT suggested no tumor stem cells.

Of quite interest is that the tumor cells were positive strongly for PSA (highly specific to prostate) and AMACR (relatively specific to prostate). Also of interest, myoepithelial cells were noted beneath the tumor cells in some minor areas, but not all areas. These myoepithelial cells could be found in IHC for HMWCK, CK14 and p63, but was not discernible in HE stainings. A retrospective view of the HE staining showed only questionable myoepithelial cells. These observations strongly suggest that the tumor (TVA indistinguishable on HE sections from TVA of GI) might originate from prostatic gland (ductal or acinar) epithelium in the PU. The positive PSA and AMACR and negative myoepithelium in the present tumor suggest that the present tumor is a rare pancreatic ductal adenocarcinoma. The author did not know 
the histogenesis of villous and tubular adenomas of non-PU urinary tracts.

The above suggestions pose serious problems in pathological diagnosis. In prostatic carcinoma, the gold standard for the distinction between benign and malignant epithelium is just the presence or absence of myoepithelial cells. In the present study, myoepithelial cells were absent or interrupted in most areas of the tumor, suggesting malignancy (adenocarcinoma). However, other IHC data (such as Ki67 and p53) and histological features were at worst TVA with severe dysplasia and invasive malignancy did never occur. A strict follow-up regimen is mandatory in this patient. However, it is possible that the present case is a non-invasive prostatic ductal tubulovillous adenocarcinoma. This note causes suspicion whether low Gleason tumor of the prostate is in fact malignant or not, as is the case with latent carcinoma. Only studying the natural course of the disease might help solving the question. Since adenomas of the colon are representing precancerous lesions (multistep carcinogenesis), adenomas of the urinary tracts might undergo transformation and malignant progression as previously described..$^{[1,2]}$

\section{CONFLICTS OF INTEREST Disclosure}

The author declares no conflicts of interest.

\section{REFERENCES}

[1] Seibel JL, Prasad S, Weiss RE, et al. Villous adenoma of the urinary tract: a lesion frequently associated with malignancy. Hum Pathol. 2002; 33: 236-41. PMid: 11957151. http://dx.doi .org/10.10 53/hupa.2002.31293

[2] Kao CS, Epstein JI. Tubular adenoma of the urinary tract: a newly described entity. Hum Pathol. 2013; 44: 1890-4. PMid: 23664485 http://dx.doi.org/10.1016/j.humpath. 2013.02.017 